\title{
Veröffentlichungen von Eberhard Gönner
}

\author{
Bearbeitet von Hans-Martin Maurer
}

Im letzten Jahrgang dieser Zeitschrift erschien ein Nachruf auf Prof. Dr. Eberhard Gönner von Robert Kretzschmar ${ }^{1}$. Darin wird neben dem Wirken Gönners als leitender Archivar und zuletzt als Präsident der Landesarchivdirektion auch seine wissenschaftliche Tätigkeit auf dem Gebiet der landesgeschichtlichen Forschung betont, besonders zur Geschichte der Fürstentümer Hohenzollern und zur Siegel- und Wappenkunde. Inzwischen wurde im Nachlass Gönners eine von ihm selbst erstellte Liste seiner Veröffentlichungen entdeckt und der Schriftleitung übergeben. Sie wird im Folgenden nach den derzeitigen Richtlinien der Zeitschrift in chronologischer Ordnung vorgelegt ${ }^{2}$.

\section{Publikationen}

Die hohenzollerischen Fürstentümer. Ein geschichtlicher Rückblick zum 100. Jahrestag des Übergangs Hohenzollerns an Preußen, in: Schwäbische Heimat 1 (1950) S. 202-207

Die Revolution von 1848/49 in den hohenzollerischen Fürstentümern und deren Anschluß an Preußen (Druck der Tübinger Dissertation in: Arbeiten zur Landeskunde Hohenzollerns, Heft 2), Hechingen 1952, 245 S.

Artikel über Adelberg, Bebenhausen, Blaubeuren, Buchau, Ellwangen, Ernst von Zwiefalten, Heiligenberg, Heiligkreuztal, Hohenzollern, Königsbronn und Lorch in: Lexikon für Theologie und Kirche, Bände 1 ('1957), 2 ( $\left.{ }^{2} 1959\right), 3$ ( $\left.{ }^{2} 1961\right)$ An den Quellen der Geschichte. Zum Weggang des Leiters des Staatsarchivs Sigmaringen Oberarchivrat Dr. Herberhold, in: Schwäbische Zeitung, Ausgabe Sigmaringen, Nr. 229 vom 3.10.1957

Dr. Herberhold als geschichtlicher Forscher, in: ebd. Nr. 231 vom 5.10.1957

1 Robert Kretzschmar, Eberhard Gönner (1919-2012). Ein Nachruf, in: ZWLG 72 (2013) S. 509-514.

2 Eine überblicksartige Zusammenstellung der Publikationen Eberhard Gönners bietet auch eine Suchabfrage unter http://opac.bsz-bw.de/WPlabw.html (Abfrage: 24.1.2014). 
Wappenbuch des Landkreises Sigmaringen (Veröffentlichungen der Staatlichen Archivverwaltung Baden-Württemberg, Heft 4), Stuttgart 1958, 62 S.

Die Grafschaft Tettnang, in: Vorderösterreich, eine geschichtliche Landeskunde, hg. von Friedrich Metz, Bd. 2, Freiburg i. Br. 1959, S. 617-625

Die Landvogtei Schwaben, in: ebd. S. 654-676

Artikel über Graf Eberhard den Milden von Württemberg, Herzog Eberhard im Bart von Württemberg, Herzog Eberhard d. J. von Württemberg, Graf Eitel Friedrich von Hohenzollern, Herzog Friedrich von Württemberg, Herzog Friedrich Karl von Württemberg, in: Neue Deutsche Biographie, Bd.4, 1959, und Bd. 5, 1961

24 Artikel in: Wörterbuch zur Geschichte, hg. von Erich BAYER, Stuttgart 1960

Wappenbuch des Landkreises Böblingen (mit Ortsgeschichten von Karl Hess) (Veröffentlichungen der Staatlichen Archivverwaltung Baden-Württemberg, Heft 7), Stuttgart 1960, 111 S.

Die kommunale Heraldik in Württemberg seit 1806, in: Der Archivar 14 (1961) Sp. 363-368

Oberschwaben. Eine historische Untersuchung über Namen und Begriff, Wangen 1961. - Abdruck auch in: Ulm und Oberschwaben 36 (1962) S. 7-18

Siegel und Wappen württembergischer und hohenzollerischer Dorfgemeinden vor 1806, in: Neue Beiträge zur südwestdeutschen Landesgeschichte. Festschrift für Max Miller (VKgL B 21), Stuttgart 1962, S. 290-308

Artikel über die Siegel und Wappen der württembergischen Städte, in: Württembergisches Städtebuch, hg. von Erich KeysER, Stuttgart 1962, jeweils Absatz 12

Das Wappen der Erzherzogin Mechthild. Überraschende Entdeckung in der Holzgerlinger Kirche, in: Aus Schönbuch und Gäu (Böblinger Bote), 1962, S. 39

Der Kreisarchivar in Baden-Württemberg, in: Der Archivar 16 (1963) Sp. 69-73

Siegel und Wappen des Klosters Blaubeuren, in: Das Kloster Blaubeuren im Mittelalter, hg. von Otto-Günter LonHard (VKgL B 25), Stuttgart 1963, S. 161-171

Abschnitt „Schwaben“, in: Geschichte der deutschen Länder, „Territorien-Ploetz“, hg. von G.W. SAnte und A. G. Ploetz-Verlag, 1. Bd., Würzburg 1964, S. 291-319 Mitarbeit am Abschnitt „Schweiz“, in: ebd. S. 274-276

Wappenbuch des Stadt- und des Landkreises Heilbronn mit einer Territorialgeschichte dieses Raumes (Veröffentlichungen der Staatlichen Archivverwaltung Baden-Württremberg, Heft 9), Stuttgart 1965, 199 S.

Ortsartikel der Landkreise Böblingen und Ravensburg, in: Handbuch der Historischen Stätten Deutschlands, Bd.6: Baden-Württemberg, hg. von Max Miller (Kröners Taschenausgabe Bd.276), Stuttgart 1965

Reverenz vor der Vergangenheit (zur Grundsteinlegung des Hauptstaatsarchivs), in: Stuttgarter Zeitung Nr. 151 vom 3.7.1965, S. 33

Wappenbuch des Landkreises Göppingen (zusammen mit Heinz BARDUA) (Veröffentlichungen der Staatlichen Archivverwaltung Baden-Württemberg, Heft 13), Stuttgart 1966, 79 S. 
Ortsgeschichtliche Artikel [für Württemberg], in: Brockhaus Enzyklopädie, Bd. 1, 1966, bis Bd. 20, 1974

Die Wappenteppiche im Stuttgarter Neuen Schloß, in: Beiträge zur Landeskunde, Beilage zum Staatsanzeiger, Nr.1/2 ( Juli 1966) S.11-14. - Nachdruck in: Baden-Württemberg 14 (1967), Heft 12, S. 2-4

Das Wappen des Herzogtums Schwaben und des Schwäbischen Kreises, in: ZWLG 26 (1967) S. 18-45

Siegel und Wappen der Stadt Waiblingen, in: Waiblingen in Vergangenheit und Gegenwart 2 (1967) S. 7-16

Tirol und Baden-Württemberg. Über die historischen Beziehungen - anlässlich des Besuchs der Tiroler Landesregierung, in: Staatsanzeiger für Baden-Württemberg, 17. Jg., Nr. 41/42 vom 25.5.1968, S. 1 f.

Die Heraldik in Georg Gadners Chorographia von 1596, in: Beiträge zur geschichtlichen Landeskunde - Geographie, Geschichte, Kartographie. Festgabe für Ruthardt Oehme (VKgL B 46), Stuttgart 1968, S. 96-102

Das Hauptstaatsarchiv Stuttgart. Seine Bestände und Aufgaben. Hg. von der Archivdirektion Stuttgart, Stuttgart 1969

Neue Aufteilung der Bestände zwischen dem Hauptstaatsarchiv Stuttgart und dem Staatsarchiv Ludwigsburg, in: Der Archivar 22 (1969) Sp. 419-421

Warum Ortsgeschichte? in: Stadt Sindelfingen, Jahresbericht 1968, 1969, S. 313-317

Neuerscheinungen zur Geschichte Württembergs und Hohenzollerns 1965-1968 (Sammelbericht), in: Blätter für deutsche Landesgeschichte 105 (1969) S. 401-427

Hauptstaatsarchiv Stuttgart und Staatsarchiv Ludwigsburg, in: Archivum 15 (1965), Paris 1969, S. 33-38

Wappenbuch des Landkreises Waiblingen (zusammen mit Heinz BARDUA) (Veröffentlichungen der Staatlichen Archivverwaltung Baden-Württemberg, Heft 24), Stuttgart 1970, 109 S.

Beiträge zu: Geschichte der deutschen Länder, „Territorien-Ploetz“, 2. Bd.: Die deutschen Länder vom Wiener Kongreß bis zur Gegenwart, hg. von Georg Wilhelm Sante und A. G. Ploetz-Verlag, Würzburg 1971: Das Königreich Württemberg S. 408-445, Hohenzollern S. 446-447, Das Land Württemberg-Hohenzollern S.729-735, Die Entstehung des Südweststaates S.735-739, Das Land Baden-Württemberg S. 739-753

Wappenbuch des Landkreises Wangen (zusammen mit Heinz BARDuA) (Veröffentlichungen der Staatlichen Archivverwaltung Baden-Württemberg, Heft 27), Stuttgart 1972, 95 S.

Kommunale Heraldik in Baden-Württemberg, in: Beiträge zur Landeskunde, Beilage zum Staatsanzeiger, Nr. 6 (Dez. 1972) S. 1 -7

Geschichte des Kreises [Hechingen] - ein Stück hohenzollerischer Geschichte, in: Abschied vom Landkreis Hechingen. Letzte Sitzung des Kreistags am 15. Dezember 1972 auf der Burg Hohenzollern, hg. vom Landkreis, Hechingen 1973, S. 19-38 
Wappen - Symbole der Geschichte. Ausstellung im Hauptstaatsarchiv Stuttgart, Katalog unter Mitarbeit von Heinz Bardua, Günter Cordes und Rainer TRunk, Stuttgart 1973, 80 S.

Der württembergische Raum und Vorarlberg, in : Montfort, Heft 2/3 (1973) S. 182188

Ansprache bei der Feier zum vierzigjährigen Bestehen des Heimatvereins Waiblingen, in: Heimatverein Waiblingen, Geschichts- und Altertumsverein, Waiblingen 1974, S. 26-29

Baden-Württemberg. Geschichte seiner Länder und Territorien (zusammen mit Günther Haselier), Würzburg 1975, 182 S. (2. ergänzte Aufl. 1980)

Max Miller zum Gedenken, in: Der Archivar 29 (1976) Sp. 157-164

Kommunale Siegel und Wappen im ehemaligen Landkreis Hechingen, in: Zeitschrift für Hohenzollerische Geschichte 12 (1976) S. 123-158

Das Wappen des Hohenzollerischen Landeskommunalverbandes, in: ebd. S. 159160

Berichte über die Sitzungen des internationalen Kommitees für Siegelkunde 19771984, in: Der Archivar 30 (1977) - 38 (1985)

Das Land Baden-Württemberg seit 1952, in: Schulzeit. Schulzeitung des Kultusministeriums Nr. 1 (1977) S. 11-14

Urkunden, in: Die Zeit der Staufer. Geschichte - Kunst - Kultur. Katalog der Ausstellung, Bd. I, Stuttgart 1977, S. 1-16, 237-241

Fürstenerziehung im 19. Jahrhundert. Die Erziehung des Prinzen Karl Anton von Hohenzollern-Sigmaringen, in: Landesgeschichte und Geistesgeschichte. Festschrift für Otto Herding (VKgL B 92), Stuttgart 1977, S. 419-441

Schillerpreis-Träger Paul Sauer, in: Geschichtsbewußtsein statt Geschichtsflucht. Schillerpreis der Stadt Marbach 1977, Ulm 1978, S. 3-9

Hohenzollern und Württemberg, in: Bausteine zur geschichtlichen Landeskunde von Baden-Württemberg, hg. von der Kommission für geschichtliche Landeskunde in Baden-Württemberg, Stuttgart 1979, S. 239-254

Neuerscheinungen zur Geschichte Württembergs und Hohenzollerns 1969-1975 (Sammelbericht), in: Blätter für deutsche Landesgeschichte 115 (1979) S. 390-410 Unsere Geschichte - Last und Hilfe, in: Ellwanger Jahrbuch 28 (1979/80) S. 7-22

Die hohenzollerischen Stände, in: Von der Ständeversammlung zum demokratischen Parlament, hg. von der Landeszentrale für politische Bildung, Stuttgart 1982, S. 183-201

Der „Münsinger Vertrag“ [1482], in: Münsingen. Geschichte, Landschaft, Kultur, hg. von der Stadt Münsingen, Sigmaringen 1982, S. 13-30

Hechingen - Aus der Geschichte einer Residenzstadt, in: ... lebenslang zu allen Stunden mit unserer Zollerheimat eng verbunden. Reden zu Ehren von Willy Baur aus Anlaß seines 85. Geburtstags, hg. vom Schwäbischen Heimatbund, Stuttgart 1982, S. 19-35

Erich Maschke, 1900-1982, Nachruf, in: ZGO 130 (1982) S. 363-365 
Zum 80. Geburtstag von Werner Fleischhauer, in: ZWLG 42 (1983) S. 3 f.

König Karl (1864-1891), in: 900 Jahre Haus Württemberg, hg. von Robert UHLAND, Stuttgart 1984, S. 328-340

König Wilhelm II. (1891-1918), in: ebd. S. 341-362

Grußwort [zum 50-jährigen Jubiläum des Heimatvereins Waiblingen 1984], in: Waiblingen in Vergangenheit und Gegenwart 8 (1986) S. 48-50

Historische Besinnung als Weg zur Selbsterkenntnis [Festvortrag zur 1200-JahrFeier Hechingens], in: Hohenzollerische Zeitung Nr. 108 vom 13.5.1986

Hechingen in preußischer Zeit. Vortrag zur 1200-Jahr-Feier, in: ebd. Nr. 217 vom 20.9.1986

Der Blaubeurer Vertrag von 1516, in: Blaubeuren. Die Entwicklung einer Siedlung in Südwestdeutschland, hg. von Hansmartin Decker-Hauff und Immo EberL, Sigmaringen 1986, S. 245-263

1200 Jahre Hechingen [Vortrag vom 18. April 1986], in: 1200 Jahre Hechingen. Beiträge zur Geschichte, Kunst und Kultur der Stadt Hechingen, Hechingen 1987, S. 25-36

Hechingen in preußischer Zeit, in: ebd. S. 99-116

Landesgeschichtliche Vereinigungen in Baden-Württemberg. Bearb. im Auftrag der Kommission für geschichtliche Landeskunde in Baden-Württemberg, Stuttgart 1987, 141 S. - Zweite vermehrte und aktualisierte Aufl. Stuttgart 1999, 262 S.

Einführung in: Die Kreis- und Gemeindewappen im Regierungsbezirk Stuttgart, bearb. von Heinz BARDUA, hg. von der Landesarchivdirektion Baden-Württemberg, Bd. 1, 1987, S. 9-14. - Desgleichen in: Bände 2-4 für die Regierungsbezirke Karlsruhe, bearb. von Herwig JoHn, 1990; Freiburg, bearb. von Herwig JoHN und Martina Heine, 1989; Tübingen, bearb. von Heinz Bardua, 1987

Ministerpräsident Karl von Weizsäcker, in: ZWLG 48 (1989) S. 359-374

Johann Adam Kraus 85 Jahre alt, in: Hohenzollerische Heimat 39, Nr.2 (1989) S. $17 \mathrm{f}$.

Die Gründung des Marienhospitals und die Entwicklung bis 1918, in: 100 Jahre Marienhospital Stuttgart, hg. von Margarita BEITL, Untermarchtal 1990, S. $23-$ 48

Die Geschichte der Kirche und des Klosters, in: St. Luzen in Hechingen, hg. von Hans-Jörg Mauser und Rudolf Schatz, Stuttgart 1991, S. 9-48

Dr. Freiherr von Ruepprecht zum Abschied, in: Südwestdeutsche Blätter für Familien- und Wappenkunde 20 (1991) S. $1 \mathrm{f}$.

Hohenzollern 1800 bis 1918, in: Handbuch der baden-württembergischen Geschichte, Bd. 3, hg. von Hansmartin Schwarzmaier, Stuttgart 1992, S. 433-475

Die St. Ulrichskapelle in Neckarhausen, in: Glatter Schriften 5 (1991) S. 111-157

Robert Uhland. Ein Nachruf, in: Suevica. Beiträge zur schwäbischen Literaturund Geistesgeschichte 6 (1991) S. 107-112 
„Versunken und vergessen“ - das Arbeitsdienstlager im Krähloh, in: Allgäuschau Leutkirch. Messebeilage der Schwäbischen Zeitung, Lokalausgabe Leutkirch, 4. Juni 1993

Hohenzollern und Württemberg. Geschichtliche Kräfte im Zollernalbkreis, in: Zollernalb-Profile. 20 Jahre Zollernalbkreis. Jahrbuch des Kreises, Bd. 3, Balingen 1993, S. 9-26

Reitersiegel in Südwestdeutschland, in: Aus südwestdeutscher Geschichte. Festschrift für Hans-Martin Maurer, hg. von Wolfgang Schmierer, Günter Cordes, Rudolf Kiess und Gerhard Taddey, Stuttgart 1994, S. 151-167

Die historischen und denkmalpflegerischen Bestrebungen der Freiherren von $\mathrm{O}_{\mathrm{w}}$ im 19. Jahrhundert, in: Adel am oberen Neckar. Beiträge zum 900jährigen Jubiläum der Familie von $\mathrm{Ow}$, hg. von Franz Quarthal und Gerhard Faix, Tübingen 1995, S. 513-539

Die Revolution von 1848/49 in den Hohenzollerischen Fürstentümern und der Übergang an Preußen, in: Hohenzollern (Schriften zur politischen Landeskunde Baden-Württemberg, Bd.23), hg. von Fritz Kallenberg, Stuttgart 1996, S. 283-306. - Darin auch: Josef Blumenstetter, S. 460-466; Josef Sprißler, S. 466472; Carl Otto Würth, S. 472-476

\section{Schriftleitung und Herausgebertätigkeit}

Zeitschrift für Württembergische Landesgeschichte, Jahrgang 33 (1974) bis Jahrgang 37 (1978)

Herausgabe des 6. und 7. Bandes der Reihe „Lebendige Vergangenheit“, Schriftenreihe des Württembergischen Geschichts- und Altertumsvereins:

Ein liberaler Theologe und Schulmann in Württemberg. Erinnerungen von Dr. Gustav v. Binder 1807-1885, hg. von Max Neunhöffer (= Lebendige Vergangenheit, Bd.6), Stuttgart 1975, $190 \mathrm{~S}$.

Theodor Pfizer: Im Schatten der Zeit. 1904-1948 (= Lebendige Vergangenheit, Bd. 7), Stuttgart 1979, 188 S. 